\title{
El sistema de financiación de las CC.AA. de régimen común y la desigualdad de la recaudación regional (1986-2007)
}

\author{
JUAN DE DIOS JIMÉNEZ AGUILERA, ROBERTO MONTERO GRANADOS, \\ PEDRO E. BARRILAO GONZÁLEZ y ELENA VILLAR RUBIO \\ Departamento de Economía Aplicada \\ UNIVERSIDAD DE GRANADA \\ e-mail: juande@ugr.es; montero@ugr.es; pedroe@ugr.es; elvillar@ugr.es
}

\begin{abstract}
RESUMEN
La reforma del acuerdo de financiación de las CC.AA. españolas de 2009, en vigor desde enero de 2010, profundiza en la corresponsabilidad fiscal, ampliando la capacidad normativa de algunos impuestos y la participación en las cuotas territorializadas del Impuesto sobre la Renta de las Personas Físicas (IRPF), el Impuesto sobre el Valor añadido (IVA) y algunos Impuestos Especiales (IIEE). Según el mencionado acuerdo, el porcentaje descentralizado de la recaudación por IVA y por impuestos especiales se distribuye entre las Comunidades Autónomas (CC.AA.) en función de índices de consumo territorializado mientras que la recaudación por IRPF se distribuye en función de la cuota íntegra declarada por los sujetos pasivos residentes en cada Comunidad Autónoma (C.A.). Este artículo pretende mostrar evidencia sobre la traslación del ingreso tributario entre regiones en los tres tributos debida, en parte, a que el ingreso se realiza en la C.A. del domicilio fiscal del sujeto pasivo independientemente de donde se produzca el hecho imponible. Las conclusiones justifican la distribución del acuerdo por IVA e IIEE en función de criterios basados en el consumo territorializado, pero no así la distribución del tramo autonómico por IRPF que se distribuye en función del ingreso de los residentes.
\end{abstract}

Palabras clave: Descentralización, traslación tributaria interterritorial, índices de concentración, equidad y eficiencia en la recaudación.

\section{Financing Agreement for the Spanish Regions and Regional Inequality by Tax Collection (1986-2007)}

\begin{abstract}
Since January, 2010, there's a new public financing agreement for the Spanish regions. This is based on the fiscal responsibility, expanding the regulatory capacity of some taxes and increasing the territorialized participation in the Income Tax (PIT), values added tax (VAT) and some special taxes (IISS). According to that agreement, the revenues decentralized of VAT and excise duties are distributed among the Autonomous Communities (CC.AA.) in terms of consumption rates, while the personal income tax revenue is distributed according to the amount declared by the persons living in each Autonomous Community (C.A.). This article treat on transfer of tax revenue between regions in the three taxes, due primarily that the tax pay is made in the C.A. of taxpayer's residence regardless of where the taxable event occurs. The findings justify the income distribution for IISS and VAT based on territorialized consumption, but not the regional distribution of personal income tax which is distributed according to revenue of residents.
\end{abstract}

Keywords: Decentralization, Interregional Income Displacement, Concentration Indices, Equity and Efficiency in Tax Collection.

Clasificación JEL: H72, H73.

Artículo recibido en febrero de 2010 y aceptado en mayo de 2010.

Artículo disponible en versión electrónica en la página www.revista-eea.net, ref. ə-28114. 


\section{INTRODUCCIÓN}

El Acuerdo 6/2009 del Consejo de Política Fiscal y Financiera de 15 de julio para la reforma del sistema de financiación de las Comunidades Autónomas de régimen común y Ciudades con Estatuto de Autonomía, aprobado el 17 de diciembre de 2009 por el Congreso de los Diputados, supone una reforma del anterior acuerdo de financiación, en vigor desde enero de 2002 (Ley 21/2001). Uno de los ejes básicos de la reforma es dotar de un aumento de "Autonomía y Corresponsabilidad" a las CC.AA. que, entre otros aspectos, el Acuerdo interpreta como un aumento de la participación de la financiación de éstas en la recaudación territorializada de algunos tributos, en la mayoría de los casos mediante un simple aumento del porcentaje de cesión.

En este sentido, se incrementa de un 33\% a un 50\% la participación en el rendimiento recaudatorio por IRPF obtenido en cada C.A. de régimen común. También se amplia de un 35\% a un 50\% la participación en la recaudación líquida por IVA y respecto de los impuestos especiales (IIEE), salvo aquellos que estaban ya completamente cedidos, se amplia la cesión de un $40 \%$ a un $58 \%$ (Cuadro I).

CUADRO I

Cesión de participación en recaudación y criterio de distribución entre CC.AA.

\begin{tabular}{|c|c|c|c|}
\hline & $\begin{array}{l}\text { Acuerdo } \\
2002(\%)\end{array}$ & $\begin{array}{l}\text { Acuerdo } \\
2009(\%)\end{array}$ & $\begin{array}{c}\text { Criterio de Distribución } \\
\text { entre CC.AA. }\end{array}$ \\
\hline IRPF & 33 & 50 & Rendimiento del Impuesto \\
\hline IVA & 35 & 50 & $\begin{array}{l}\text { Consumo certificado por el } \\
\text { INE }\end{array}$ \\
\hline Impuesto sobre la Cerveza & 40 & 58 & $\begin{array}{l}\text { Consumo certificado por el } \\
\text { INE }\end{array}$ \\
\hline $\begin{array}{l}\text { Impuesto Sobre el vino y } \\
\text { bebidas fermentadas }\end{array}$ & 100 & 100 & (Actual tipo de gravamen 0 ) \\
\hline $\begin{array}{l}\text { Impuestos sobre productos } \\
\text { intermedios, sobre alcohol y } \\
\text { bebidas derivadas }\end{array}$ & 40 & 58 & $\begin{array}{l}\text { Consumo certificado por el } \\
\text { INE }\end{array}$ \\
\hline Impuesto sobre Hidrocarburos & 40 & 58 & $\begin{array}{l}\text { Entregas de gasolina, } \\
\text { gasóleos y fuelóleos según } \\
\mathrm{M}^{\circ} \text { de Industria ponderados } \\
\text { por tipos impositivos }\end{array}$ \\
\hline $\begin{array}{l}\text { Impuesto sobre labores del } \\
\text { Tabaco }\end{array}$ & 40 & 58 & $\begin{array}{l}\text { Ventas a expendedurías } \\
\text { certificadas por Comisionado } \\
\text { para el Mercado de Tabacos }\end{array}$ \\
\hline Impuesto sobre la electricidad & 100 & 100 & $\begin{array}{l}\text { Consumo de electricidad. } \\
\text { Datos del } \mathrm{M}^{\circ} \text {. de Industria. }\end{array}$ \\
\hline
\end{tabular}

Fuente: Sistema de Financiación de las CC.AA. 
La ampliación del espacio fiscal hacia las CC.AA. tiene evidentes ventajas, sobre todo por el incremento de la denominada corresponsabilidad fiscal, lo que implica que los decisores políticos de las haciendas autonómicas tienen mayores incentivos para que sus políticas de gasto se adecuen a los ingresos que generen en sus respectivos territorios. En este sentido la teoría del federalismo fiscal recomienda que, en la medida de lo posible, los procesos de descentralización de gasto se vean acompañados de procesos paralelos de descentralización de ingresos. La literatura económica (Brandforf y Oates, 1971; Quigley y Smolnensky, 1993; Hines y Thaller, 1995; etc.) prácticamente es unánime en afirmar que cuando la descentralización de ingresos no es proporcional a la de gasto, es decir se financian con transferencias de la unidad central de gobierno y no son responsables directos de la recaudación de los tributos, los gobiernos subcentrales tienden a excederse más en el gasto público.

Sin embargo, la descentralización de ingresos también conlleva algunos inconvenientes como la reducción de la cohesión fiscal interterritorial (siquiera en términos de homogeneidad normativa tributaria) y socavan la solidaridad interterritorial de forma que las regiones más ricas pueden intentar retener y limitar las transferencias desde sus territorios hacia los territorios menos privilegiados. De los perjuicios que pueden llegar a ocasionar ambas cuestiones tenemos amplia experiencia en España con los casos de las Comunidades Forales de País Vasco y Navarra (con dificultades casi insalvables para la negociación del cupo vasco o de la aportación de Navarra y con múltiples casos de competencia fiscal desleal, alguno de los cuales ha llegado hasta los tribunales europeos) y toda la literatura sobre transferencias horizontales y balanzas fiscales ${ }^{1}$.

En este trabajo se define como recaudación efectiva territorializada la suma de los ingresos tributarios de los contribuyentes en cada región y se define como re-

\footnotetext{
${ }^{1}$ Las transferencias horizontales históricamente se han estudiado a través del mecanismo de las balanzas fiscales, instrumento de información económica en el que se explicitan los flujos fiscales habidos, en un período determinado, entre cada una de las regiones que integran un país y el resto. El dato más característico es el saldo fiscal (diferencia entre los beneficios obtenidos y los costes soportados por cada región), el cual permite la caracterización de las regiones como contribuyentes o como beneficiarias netas de la actividad financiera del Gobierno Central. Desde 1960, ha habido 36 estimaciones de balanzas fiscales llevadas a cabo por diversos autores. Entre otros: Barberán, R. (2004), Castells et al. (2000), De la Fuente (2001), Uriel (2001) y el propio Gobierno de España (2008) ha publicado, por primera vez, unas balanzas fiscales de las CC.AA. con el Sector Público Estatal. Se fundamentan en dos metodologías básicas: "Metodología carga-beneficio", en la que los ingresos se imputan al territorio donde residen los ciudadanos, mientras que los gastos se imputan al territorio en el que residen las personas a las que van destinados los servicios públicos o las transferencias públicas; y "Metodología del flujo monetario", en la que los ingresos se imputan al territorio en el que se localiza la capacidad económica sometida a gravamen y los gastos al territorio donde tienen lugar. En la mayoría de los estudios, las balanzas fiscales se aproximan a transferencias de recursos desde las CC.AA. con mayor potencial económico hacia aquellas otras consideradas más pobres; las CC.AA. con mayor renta per cápita son las que más contribuyen, presentando un défícit fiscal y, por el contrario, aquellas con menor renta per cápita obtienen superávit fiscal; asociando estos traspasos a motivos de equidad y solidaridad, y dejando en un segundo plano el motivo de la eficiencia.
} 
caudación potencial territorializada la que debería haberse recaudado en cada región de acuerdo con las variables macroeconómicas (renta, consumo, etc.) que forman parte del hecho imponible de cada uno de los tres impuestos. Veremos que ambas nunca coinciden, entre otras circunstancias porque no todos los tributos se ingresan en el lugar en el que se produce el hecho imponible de los mismos, sino que éste se realiza, según la normativa de los impuestos, en el lugar de residencia del sujeto pasivo. Así, por ejemplo, el IVA se ingresa en la región del domicilio fiscal de la empresa vendedora, configurada como sujeto pasivo según la ley formal del impuesto, independientemente de donde se produzca el consumo. Igualmente, el incremento de patrimonio derivado de la venta un inmueble se liquida en la región de residencia del sujeto pasivo independientemente de la región donde esté situada la finca.

Esto implica que existen dos tipos de transferencias compensadoras: las que buscan la equidad (un flujo desde las CC.AA. ricas hacia las CC.AA. pobres) y las que buscan la justicia-eficiencia (un flujo desde las CC.AA. que atraen más sujetos pasivos hacia aquellas en que se produce el hecho imponible). Supondremos, por la experiencia en España, que las transferencias compensadoras (sean por la eficiencia o por la equidad) son muy difíciles de gestionar e introducen tensiones y rigideces en el sistema.

En este sentido, el Acuerdo de 2009 contiene, a nuestros efectos, tres elementos clave: a) la necesidad de cada C.A. $\left(N_{i}\right)$; los recursos territoriales de los tributos $\left(T_{i}\right)$ y las entregas del fondo de suficiencia $\left(S_{i}\right)$ como mecanismo de cierre. De forma que para cada C.A. ( $i)$ se cumple que $S_{i}=N_{i}-T_{i}$. Es decir sólo contempla un tipo de transferencia (por la equidad). En cualquier caso cada C.A. recibe los recursos que necesita pero no es estrictamente igual que éstos se obtengan mediante transferencias por la recaudación territorializada que mediante transferencias de equidad. Así, por ejemplo considérese el siguiente caso: Una economía descentralizada formada por dos regiones con igual renta y riqueza pero que la mayoría de los ingresos tributarios se realizan en una C.A. por el mero hecho de que las empresas están domiciliadas allí. Entonces la otra C.A. tendrá transferencias por equidad cuando en realidad son ingresos que le corresponden en justicia, y ¿qué ocurre si se incrementa el porcentaje de tributos cedidos? pues que la injusticia aumentará porque tendrán que ser mayores las transferencias por equidad.

Entonces, si se produce una gran desigualdad entre la recaudación efectiva y la recaudación potencial de las unidades subcentrales, el grado de descentralización tributaria no podrá ser alto porque la rigidez de las transferencias compensatorias que deberían entrar en funcionamiento haría devenir al sistema en ineficiente. Esto puede suponer una limitación, dentro de la teoría de la descentralización, al máximo grado de descentralización posible en una economía. De esta forma si, en una economía, se distribuye la recaudación tributaria de forma homogénea por todo el territorio, entendiendo que se ajusta su recaudación efectiva a la potencial, el grado de descentralización fiscal podrá ser alto, pero si la recaudación se distribuye de forma asimétrica entre las distintas regiones el grado de descentralización fiscal 
deberá ser inferior porque, de lo contrario, se generalizaría la necesidad de transferencias horizontales. El grado de homogeneidad de la recaudación constituye, por tanto, un indicador del grado de descentralización fiscal que puede soportar una economía.

En este sentido cabe mencionar que, en el actual sistema de financiación español (Acuerdo 2009) la distribución de recursos tributarios hacia las CC.AA. se realiza, tanto en el caso del IVA como de los IIEE, en función de índices de consumos territorializados (no de ingresos). Sin embargo, en el caso del IRPF se realiza en función del "rendimiento del impuesto" un vago concepto que sí que alude a la cuota íntegra autonómica recaudada efectivamente en cada C.A.

El objetivo de este artículo será cuantificar la recaudación efectiva realizada en cada C.A. y compararla, durante una serie de años con la recaudación potencial que le correspondería en función de las variables macroeconómicas que recogen los distintos hechos imponibles. La comparación se realizará mediante la construcción de dos índices de concentración para cada impuesto. La diferencia entre ambos puede considerarse como una medida del grado de desigualdad en la distribución del ingreso tributario.

Además de esta introducción, el trabajo se divide en cuatro partes, la primera describen la metodología, la segunda y tercera describe los resultados y en la última se recogen las conclusiones.

\section{METODOLOGÍA}

Se ha elaborado un panel de datos de las CC.AA. españolas que recoge la evolución dinámica de la recaudación de los tres tributos parcialmente descentralizados en el actual Modelo de Financiación (Impuesto sobre la Renta de las Personas Físicas (IRPF), Impuesto sobre el Valor Añadido (IVA) e Impuestos especiales (IIEE)) y las variables macroeconómicas relevantes que constituyen sus respectivas Bases Imponibles para un período comprendido desde 1986 a 2007 en el caso del IRPF e IVA y de 2000 a 2005 en el caso de los IIEE. Los años de inicio y fin están determinados por la disponibilidad de datos tanto de recaudación como macroeconómicos. Los datos de recaudación se han obtenido de las Memorias Anuales de Recaudación de Tributos de la Agencia Estatal de la Administración Tributaria (AEAT) salvo la recaudación territorializada del Impuesto sobre Transmisiones Patrimoniales que se obtuvo de la base de datos del Instituto de Estudios Fiscales (BADES$\mathrm{PE}$ ), mientras que los datos macroeconómicos se han obtenido de las bases de datos de la Contabilidad Regional de España (base 2000: CRE-00 y base 1986. CRE86) disponibles en www.ine.es. Por su especial régimen fiscal se ha excluido a Canarias del modelo de IRPF e IVA y al País Vasco, Navarra, Ceuta y Melilla de los tres modelos.

La selección de las variables independientes (datos macroeconómicos regionales) se debe a criterios estrictamente normativos derivados de la legislación de cada 
impuesto. Las variables que componen el panel son las siguientes (todas ellas transformadas en sus correspondientes logaritmos naturales):

- irpf: Recaudación territorializada (por CC.AA.) por el Impuesto sobre la Renta de las Personas Físicas (residentes y no residentes).

- iva: Recaudación territorializada por el Impuesto sobre el Valor Añadido.

- iiee: Recaudación territorializada por la suma de los impuestos especiales de Alcohol y bebidas derivadas, Cerveza, Productos intermedios, Hidrocarburos, Labores del Tabaco, Determinados medios del transporte y Electricidad.

- remun: Remuneración de asalariados.

- rentas: Rentas de la propiedad mobiliaria e inmobiliaria.

- eeb: Excedente de explotación bruto y renta mixta bruta. Beneficios empresariales y los beneficios de las explotaciones familiares.

- tp: Recaudación territorializada por el Impuesto sobre Transmisiones Patrimoniales (ITP). Dicho impuesto grava las transmisiones patrimoniales onerosas entre particulares.

- cons_terr: Consumo final de los hogares (no incluido el consumo público ni de instituciones sin fines de lucro por no disponer de series homogéneas) sobre el territorio económico.

- cons_terr_IIEE: Consumo final de los hogares en los bienes clasificados en los epígrafes 02 y 04 y 07 del COICOP (EP02: Bebidas Alcohólicas, Tabaco y Narcóticos, EP04: Vivienda, Agua, Electricidad, Gas y otros Combustibles; EP07: Compra de Vehículos, Utilización de Vehículos personales y Servicios de transporte). Se han utilizado dichos epígrafes por ser los que recogen el hecho imponible de los IIEE de una forma más concreta. No obstante se han realizado distintas modelizaciones retirando uno o dos de los epígrafes con resultados muy similares. De esta variable sólo se dispone de datos desagregados desde 2000 a 2005.

- dummy: Es una variable dicotómica que recogerá, en el modelo econométrico para el IRPF, la heterogeneidad del cambio de base desde la CRE-86 y la CRE-2000. En el modelo del IVA no ha resultado significativa.

La recaudación efectiva está determinada por la recaudación territorializada en cada territorio que se ha obtenido de las Memorias de la Agencia Tributaria. La recaudación potencial de cada C.A. se realiza mediante una estimación. En el caso del IRPF y del IVA, dado que se dispone de suficientes grados de libertad debido al amplio rango de fechas tanto de las fuentes de renta normativas como de consumo territorializado, se ha utilizado análisis de regresión para panel según los siguientes modelos lineales:

$$
\begin{gathered}
i r p f_{i t}=\beta_{\mathrm{o}}+\beta_{1} \text { remun }_{i t}+\beta_{2} \text { rentas }_{i t}+\beta_{3} \text { eeb }_{i t}+\beta_{4} \text { tp }_{i t}+u_{i}+e_{i t} \\
i v a_{i t}=\beta^{\prime \prime}{ }_{\mathrm{o}}+\beta^{\prime \prime}{ }_{1} \text { cons_terr }_{i t}+u_{i}+e_{i t}
\end{gathered}
$$


En el caso de los IIEE no procede realizar análisis de regresión por cuanto el limitado rango temporal (cinco años) reduce excesivamente los grados de libertad, por lo que se ha optado por calcularlo en función de su porcentaje de participación en el consumo territorializado. De forma que la recaudación potencial de cada C.A. por los IIEE es su porcentaje de participación en el consumo territorializado en los epígrafes 02, 04 y 07 del COICOP multiplicado por la recaudación.

El modelo [1] pretende explicar en cada C.A. la recaudación por IRPF a partir de las fuentes de renta recogidas normativamente en la legislación fiscal: Remuneración de los asalariados; Excedente de explotación; Rentas de la propiedad, inmobiliaria y mobiliaria; e incrementos de patrimonio, estos últimos aproximados a partir de las liquidaciones del impuesto sobre transmisiones patrimoniales. La transformación logarítmica minimiza, en gran medida, los problemas que pudieran derivarse de la progresividad del impuesto y del crecimiento de las bases y recaudación. De forma similar el modelo [2] pretende explicar la recaudación por IVA a partir del consumo territorializado.

La estimación de modelos de panel también sirve para controlar la posible fuente de heterogeneidad inobservable entre CC.AA. debido a heterogeneidad en la recaudación provocada por la progresividad del IRPF y por distintos hábitos de consumo en el caso del IVA. Aunque, para incrementar la robustez de las estimaciones y los test correspondientes, se ha utilizado la base de datos completa (desde 1986 hasta 2007), aquí sólo se presentan los datos desde 2000 hasta 2005 (IVA) o 2007 (IRPF) para homogeneizar la presentación con la otra figura impositiva analizada (IIEE).

Las regresiones lineales para datos de panel pueden estimarse, entre otros, mediante tres procedimientos alternativos: a) con la base de datos completa (pooled); b) con métodos para panel de efectos fijos; y c) con métodos para panel de efectos variables. Para descartar el primero se ha realizado el test de Breusch-Pagan (1979). Para discriminar entre los dos segundos se ha realizado el test de Hausman (1978).

La recaudación potencial $\left(\hat{\tau}_{p, i t}\right)$ se ha obtenido en función de la siguiente expresión:

$$
\hat{\tau}_{p, i t}=\frac{\hat{y}_{i t}}{\sum_{i=0}^{17} \hat{y}_{i t}} \sum_{i=0}^{17} \tau_{e, i t}
$$

Donde $\tau_{e, i t}$ es la recaudación efectiva (observada) en la C.A. $i$ durante el ejercicio $t$ e $\hat{y}_{i t}$ es, para el caso de IIEE, el consumo territorializado en los epígrafes 02 , 04 y 07 del COICOP y, en el caso del IVA e IRPF, son los valores previstos por los modelos econométricos. La expresión [3] permite generar un valor esperado para cada C.A. y año como un porcentaje de participación en la recaudación efectiva de cada año.

Para el cálculo de un índice de desigualdad tributaria, se ha seguido la metodología propuesta por Kakwani, Wagstaff y van Doorslaer (Kakwani et al. 1997; 
Wagstaff et al. 1999 y van Doorsaler et al. 2000a y 2000b). De forma que se han estimado dos índices de concentración para cada impuesto y para cada año de la muestra, uno de la recaudación efectiva per cápita $\left(I C_{e}\right)$ y otro de la recaudación potencial per cápita $\left(I C_{p}\right)$. Aunque ambos están transformados en términos per cápita los cálculos recogen la heterogeneidad poblacional mediante la correspondiente ponderación.

En esta forma $I C_{e}$, que mide dos veces el área entre la curva de Lorenz de la recaudación efectiva $\left(L_{e}\right)$ y la recta de equidistribución y, por su parte $I C_{p}$ hace lo propio entre la curva de Lorenz de la recaudación potencial $\left(L_{p}\right)$ y la misma recta. $I C_{e}$ es un indicador de desigualdad, que nos dice cuánto de diferente es la recaudación entre unas regiones y otras, en un determinado país. Sin embargo, dado que parte de las diferencias en la recaudación son debidas a un distinto potencial económico: distinta riqueza, renta y consumo entre unas regiones y otras, la diferencia entre ambos índices, que denominamos como el índice de desigualdad tributaria $\left(T I=I C_{e}-I C_{p}\right)$, es una medida de las diferencias en recaudación que no son debidas a la distinta riqueza de las CC.AA. sino a la traslación de la carga tributaria por otros motivos como la domiciliación de sujetos pasivos en CC.AA. distintas de las que se produce el hecho imponible. Gráficamente sería el área sombreada de la ILUSTRACIÓN 1.

\section{ILUSTRACIÓN 1}

Curvas de Lorenz de Recaudación efectiva y Potencial.

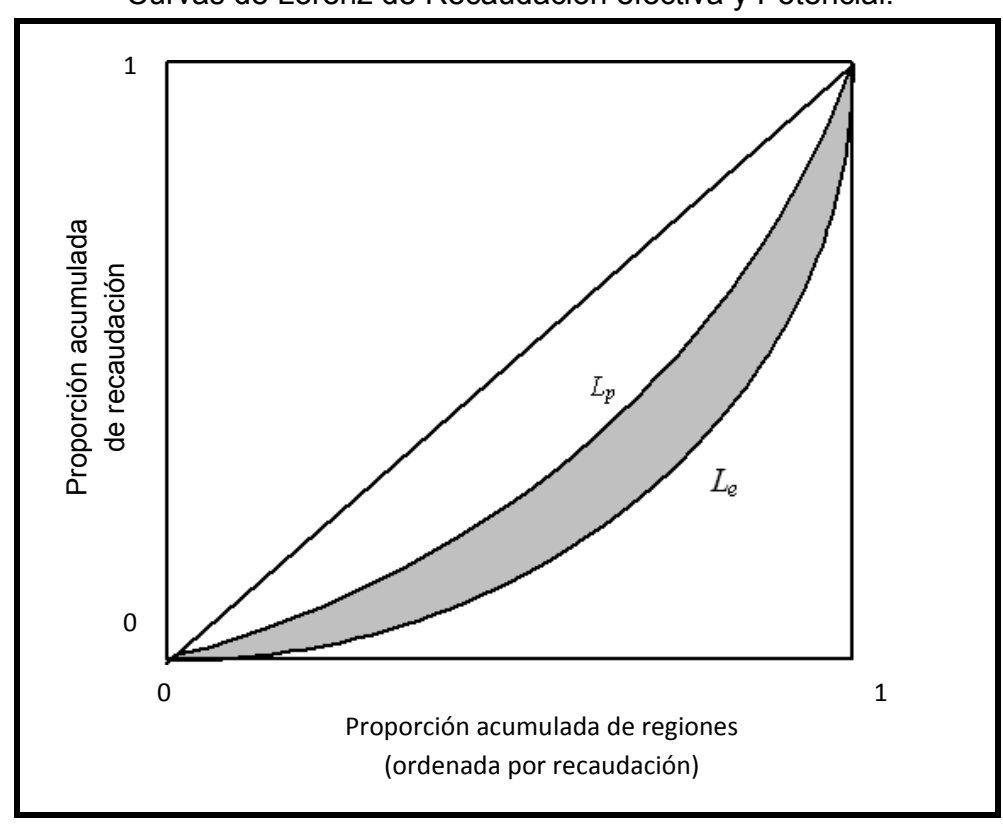


Existen muchas formas de medir los índices de concentración. En este caso y por el carácter agrupado (en CC.AA.) de los datos de recaudación hemos utilizado la expresión (Kakwani et al., 1997):

$$
I C_{e}=\frac{2}{\bar{\tau}_{e}} \sum_{i=1}^{n} f_{i}\left(\tau_{e i}-\bar{\tau}_{e}\right)\left(R_{i}-\frac{1}{2}\right)
$$

Donde $f_{i}$ es la población relativa de cada C.A., $\tau_{e . i}$ es la recaudación efectiva $(e)$ per cápita en cada región $i ; \bar{\tau}_{e}=\sum_{i=1}^{n} f_{i} \tau_{e, i}$ es el promedio ponderado de recaudación per capita de todas las CC.AA.; $R_{i}=\sum_{i=0}^{i-1} f_{i}+\frac{1}{2} f_{i}$ es el orden fraccional relativo de la región $i\left(0 \leq R_{i} \leq 1\right)$ (es decir, el porcentaje acumulado de población que queda por encima de la mediana de cada intervalo una vez que las regiones quedan ordenadas en función de la recaudación per cápita) y $\sigma_{R}^{2}$ es la varianza de $R_{i}$. De forma similar se puede obtener un índice de concentración $\left(I C_{p}\right)$ de la recaudación potencial por cada impuesto considerado.

Por su parte, del índice de desigualdad tributaria se puede calcular por diferencia entre los anteriores. El índice de desigualdad tributaria $(T I)$ puede ser considerado también como una medida del volumen de transferencias compensadoras necesarias para equiparar la recaudación efectiva de cada C.A. con su recaudación potencial. En tanto que el $I C_{p}$ puede ser considerado como una medida de la necesidad de transferencias para equiparar el ingreso en función del distinto potencial económico de las CC.AA. Es decir una medida de la necesidad de solidaridad interterritorial.

\section{RESULTADOS}

\subsection{Modelo 1: Recaudación por el IRPF}

Los test de raíz unitaria, que deben interpretarse con precaución debido al limitado número de observaciones $(n=22)$, muestran que tanto las variables dependientes como independientes son no estacionarias (crecen en el tiempo), pero que las series están cointegradas. Las estimaciones son, por tanto, no espurias ${ }^{2}$ (Resultados en anexo).

El test de especificación valora mejor los modelos de panel que la regresión lineal pooled. El modelo de efectos fijos es el más recomendable para el modelo de

\footnotetext{
${ }^{2}$ Se suelen decir que, en caso de cointegración, los estimadores lineales son superconsistentes porque convergen al verdadero valor del parámetro con una menor masa muestral.
} 
recaudación de IRPF (Resultados en el anexo). Los resultados generales del modelo econométrico funcional son (CUADRO 2).

\section{CUADRO 2}

Parámetros estimados sobre la recaudación del IRPF.

\begin{tabular}{|l|c|c|}
\hline \multicolumn{1}{|c|}{ Variable } & estimación & p-valor \\
\hline remun & 0,527 & 0,000 \\
rentas & 0,386 & 0,000 \\
tp & 0,045 & 0,375 \\
eeb & 0,341 & 0,000 \\
dummy00 & $-0,137$ & 0,000 \\
constante & $-3,625$ & 0,000 \\
ajuste $\mathrm{R}^{2}:$ & intragrupos: $94,36 \%$ & \\
& entregrupos: $91,60 \%$ & \\
Wald $\mathrm{X}^{2}$ & general: $91,90 \%$ & \\
$\mathrm{~N}:$ & 753,64 & 0,000 \\
grupos: & 308 & \\
\hline
\end{tabular}

El ajuste general del modelo es muy alto, el coeficiente de determinación general supera el 91\% de ajuste; por su parte la significación de las variables incluidas en el modelo también es alta, salvo el caso del impuesto sobre Transmisiones Patrimoniales, aunque dada la alta colinealidad de las variables independientes, y por lo tanto la alta probabilidad de alteración de los errores estándar, se ha mantenido dentro del modelo. Dado que se ha construido una relación de elasticidades la interpretación de los coeficientes es inmediata, el modelo de regresión permite afirmar, por ejemplo, que un incremento del $1 \%$ por ciento en la remuneración de los asalariados aumenta la recaudación del tributo en un $0,53 \%$. 
CUADRO 3

Recaudación EFECTIVA y potencial por el IRPF por CC.AA., 2007 (millones de euros).

\begin{tabular}{|l|r|r|r|r|}
\hline & $\begin{array}{c}\text { Recaudación } \\
\text { efectiva }\end{array}$ & $\begin{array}{c}\text { Recaudación } \\
\text { potencial }\end{array}$ & $\begin{array}{c}\text { \% Diferencia } \\
\mathbf{2 0 0 7}\end{array}$ & $\begin{array}{c}\text { \% Diferencia } \\
\text { promedio 00-07 }\end{array}$ \\
\hline Andalucía & $6.700,9$ & $11.052,6$ & 60,63 & 59,14 \\
\hline Aragón & $1.939,1$ & $1.827,9$ & 106,09 & 104,41 \\
\hline Asturias & $1.107,7$ & $1.109,3$ & 99,85 & 114,85 \\
\hline Baleares & $1.587,6$ & $1.312,5$ & 120,97 & 120,15 \\
\hline Cantabria & $1.556,2$ & 566,4 & 274,74 & 278,85 \\
\hline Castilla-LM & $1.571,8$ & $2.051,1$ & 76,63 & 69,14 \\
\hline Castilla-L & $2.392,3$ & $3.605,7$ & 66,35 & 65,40 \\
\hline Cataluña & $15.677,8$ & $18.034,5$ & 86,93 & 89,19 \\
\hline Valencia & $6.124,2$ & $7.946,3$ & 77,07 & 75,60 \\
\hline Extremadura & 665,1 & 745,3 & 89,25 & 86,36 \\
\hline Galicia & $2.724,4$ & $3.088,2$ & 88,22 & 90,26 \\
\hline Madrid & $25.848,3$ & $16.653,4$ & 155,21 & 154,43 \\
\hline Murcia & $1.312,9$ & $1.309,1$ & 100,29 & 97,20 \\
\hline Rioja (La) & 376,2 & 281,9 & 133,42 & 128,78 \\
\hline Total & $69.584,5$ & $69.584,5$ & & \\
\hline
\end{tabular}

Los resultados que, como una muestra para el año 2007, se recogen en el CUADRO 3, permiten observar importantes diferencias entre la recaudación efectiva y la potencial en cada región para 2007 (ILUSTRACIÓN 2). Aunque en valores absolutos la mayor diferencia se produce en Madrid, que recauda 9.194,9 millones $€$ más de lo que le corresponde en términos de bases imponibles, en términos relativos es aún mayor en Cantabria (la recaudación efectiva es un $278,8 \%$ superior a la potencial). Por el lado contrario, Andalucía $(60,6 \%)$ encabeza las CC.AA. en las que la recaudación efectiva es claramente inferior a la recaudación potencial. Este porcentaje implica que, en el caso de Andalucía, se deja de recaudar impuestos sobre la renta por un importe de 4.351,7 millones $€$ que se generan en su territorio pero se ingresa por los sujetos pasivos en otros. Por tanto, esta C.A. presenta un defecto de recaudación respecto a la que le correspondería en función de sus variables económicas. La última columna recoge el promedio de dichas diferencias durante los 7 últimos años de forma que se ilustra la relativa estabilidad de las mismas. La mejor explicación de dichas diferencias es la que los ingresos fiscales se realizan en la región en que se encuentra domiciliado el sujeto pasivo. La centralidad de Madrid y la domiciliación en Cantabria de importantes empresas financieras provocan que su recaudación real supere a la potencial, en el caso de Andalucía sucede lo contrario su situación de marginalidad puede estar provocando que las empresas no suelan estar domiciliadas en su territorio. 
Similares resultados se obtienen para el resto de los años analizados. La comparación de los mismos permite apreciar una evolución dinámica desde 2000 a 2007. Esta evolución será objeto de análisis en el apartado siguiente.

\section{ILUSTRACIÓN 2}

Recaudación efectiva y potencial por el IRPF por CC.AA. (2007).

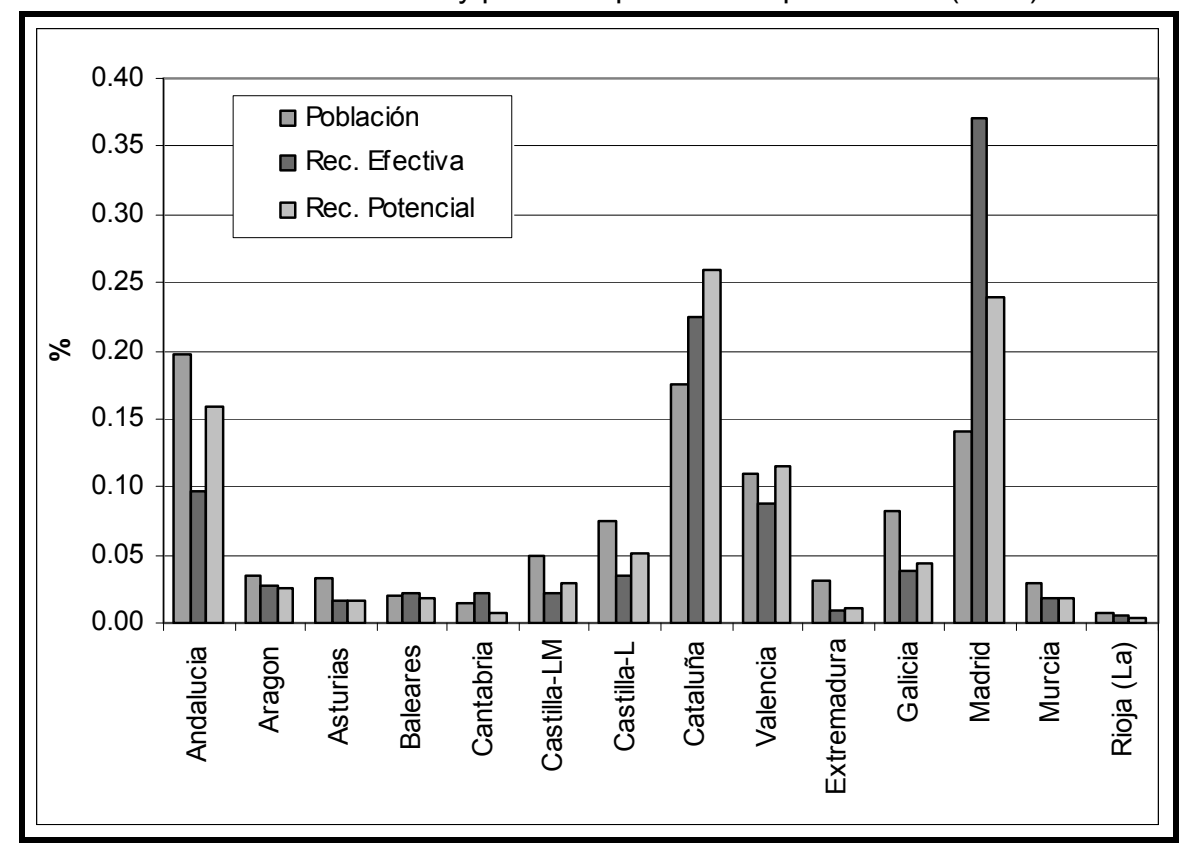

La ILUSTRACIÓN 2 muestra como por lo general el porcentaje de recaudación potencial suele ser inferior al porcentaje de población en las CC.AA. más pobres y viceversa. Sin embargo, la recaudación efectiva parece más caprichosa, concentrándose en algunas CC.AA. y reduciéndose en otras. Es decir, se está produciendo una traslación de la carga impositiva desde algunas CC.AA. hacia otras.

Además debiera tenerse en cuenta que se puede producir la traslación de la carga impositiva de este impuesto desde los sujetos pasivos hacia otros individuos. Por ejemplo un empresario afincado en una C.A. puede trasladar el impuesto sobre la renta a los residentes en otras CC.AA. mediante incrementos en los precios. Por otra parte, también es posible que las remuneraciones salariales imputadas a un territorio se hayan generado en otro, por ejemplo, el sueldo de un presidente de una empresa de ámbito nacional o de un alto funcionario está imputado en una C.A. cuando su retribución se debe a los servicios que presta en todo el territorio nacional. En la medida en que, en España, dos o tres CC.AA. tienen una evidente capacidad de atracción de sujetos pasivos en sus territorios, la traslación de carga tributaria genera beneficiarios y perjudicados netos en el sistema. La concreción de esta 
capacidad de traslación es compleja ya que requiere de una cantidad de datos de los que se carece y que obligan a recurrir a estimaciones basados en conjeturas. No obstante sí que permiten afirmar que las diferencias que aquí se recogen pueden ser, en realidad, mayores.

El importe de la suma del total de recursos que pierden las CC.AA. deficitarias, que coincide con el que ganan las excedentarias, asciende a 10.668 millones de euros anuales, lo que constituye un $15,33 \%$ del total de la recaudación.

Dado que se cede el $50 \%$ de la recaudación de este impuesto, las CC.AA. deficitarias están perdiendo un importe de 5.334 millones de euros anuales. Este importe, en la medida en que se haga homogénea la necesidad relativa de las CC.AA. quizá lo estén recuperando mediante los fondos de solidaridad, pero esa calificación les perjudica en el debate de la corresponsabilidad interterritorial.

\subsection{Recaudación por el IVA}

Al igual que en el caso del IRPF, los test de raíz unitaria muestran la no estacionariedad de las variables del modelo y la estacionariedad de los residuos ${ }^{3}$. (Resultados en anexo). Por su parte, los test de especificación valoran mejor los modelos de efectos variables para panel sobre el resto de posibilidades. (Resultados en el anexo).

La estimación de la recaudación potencial por el IVA por C.A. se basa en la proporción del consumo territorializado (cons_terr) que representa respecto del total de recaudación de las regiones consideradas. Los resultados del modelo de regresión ${ }^{4}$ [2] han sido (CUADRO 6):

\section{CUADRO 4}

Parámetros estimados sobre la recaudación del IVA.

\begin{tabular}{|l|c|c|}
\hline \multicolumn{1}{|c|}{ Variable } & Estimación & p-valor \\
\hline cons_territ & 0,976 & 0,000 \\
constante & $-2,826$ & 0,000 \\
ajuste $\mathrm{R}^{2}:$ & intragrupos: $82,3 \%$ & \\
& entregrupos: 82,7\% \\
& general: $81,8 \%$ & \\
Wald $\mathrm{X}^{2}$ & 1273,46 & 0,000 \\
$\mathrm{~N}:$ & 280 & \\
grupos: & 14 & \\
\hline
\end{tabular}

\footnotetext{
${ }^{3}$ También, al igual que en el caso del IRPF, deben interpretarse con precaución debido al tamaño muestral (22 observaciones).

${ }^{4}$ Dado que el modelo de regresión sólo contiene una variable exógena los resultados de recaudación territorializada son muy similares a los que se obtendrían mediante una distribución proporcional del consumo territorializado.
} 
El CUADRO 5, elaborado a partir de los datos de la variable dependiente y de la estimación del modelo resume las cifras de recaudación efectiva y potencial para 2005.

En dicho cuadro se refleja que son Madrid (257,5\%) y Cataluña (130,7\%) las únicas CC.AA. donde se recaudan más tributos por el concepto de IVA que el consumo que representan. En el otro extremo, se sitúan el resto de CC.AA. cuya recaudación efectiva es inferior a la potencial. En términos absolutos, la C.A. más perjudicada vuelve a ser Andalucía, en la que la recaudación efectiva es 5.207,05 millones de euros menor de la potencial. No obstante, en términos relativos, en algunas de las CC.AA. aún cuentan con ratios más desfavorables (Extremadura, Castilla-León, Castilla-La Mancha y Baleares).

\section{CUADRO 5}

Recaudación efectiva y potencial por el IVA por CC.AA., 2005 (millones de euros).

\begin{tabular}{|l|r|r|r|c|}
\hline & $\begin{array}{c}\text { Recaudación } \\
\text { efectiva }\end{array}$ & $\begin{array}{c}\text { Recaudación } \\
\text { potencial }\end{array}$ & $\begin{array}{c}\text { \% Diferen- } \\
\text { cia 2005 }\end{array}$ & $\begin{array}{c}\text { \%Diferencia } \\
\text { promedio } \\
\text { 00-05 }\end{array}$ \\
\hline Andalucía & $3.517,76$ & $8.724,81$ & 40,32 & 38,03 \\
\hline Aragón & $1.108,08$ & $1.767,75$ & 62,68 & 62,54 \\
\hline Asturias & 774,32 & $1.484,53$ & 52,16 & 48,80 \\
\hline Baleares & 609,70 & $1.549,51$ & 39,35 & 37,65 \\
\hline Cantabria & 583,86 & 746,42 & 78,22 & 75,95 \\
\hline Castilla-LM & 694,48 & $2.019,78$ & 34,38 & 36,20 \\
\hline Castilla-L & $1.058,23$ & $3.177,69$ & 33,30 & 38,35 \\
\hline Cataluña & $12.502,08$ & $9.695,53$ & 128,95 & 130,68 \\
\hline Valencia & $4.560,60$ & $5.690,80$ & 80,14 & 75,17 \\
\hline Extremadura & 227,03 & $1.155,48$ & 19,65 & 17,95 \\
\hline Galicia & $1.581,75$ & $3.395,39$ & 46,59 & 49,58 \\
\hline Madrid & $22.002,69$ & $8.703,96$ & 252,79 & 257,51 \\
\hline Murcia & 623,75 & $1.540,20$ & 40,50 & 26,27 \\
\hline Rioja (La) & 218,07 & 410,55 & 53,12 & 50,91 \\
\hline & $50.062,40$ & $50.062,40$ & & \\
\hline
\end{tabular}




\section{ILUSTRACIÓN 3}

Recaudación efectiva y potencial por el IVA por CC.AA. 2005.

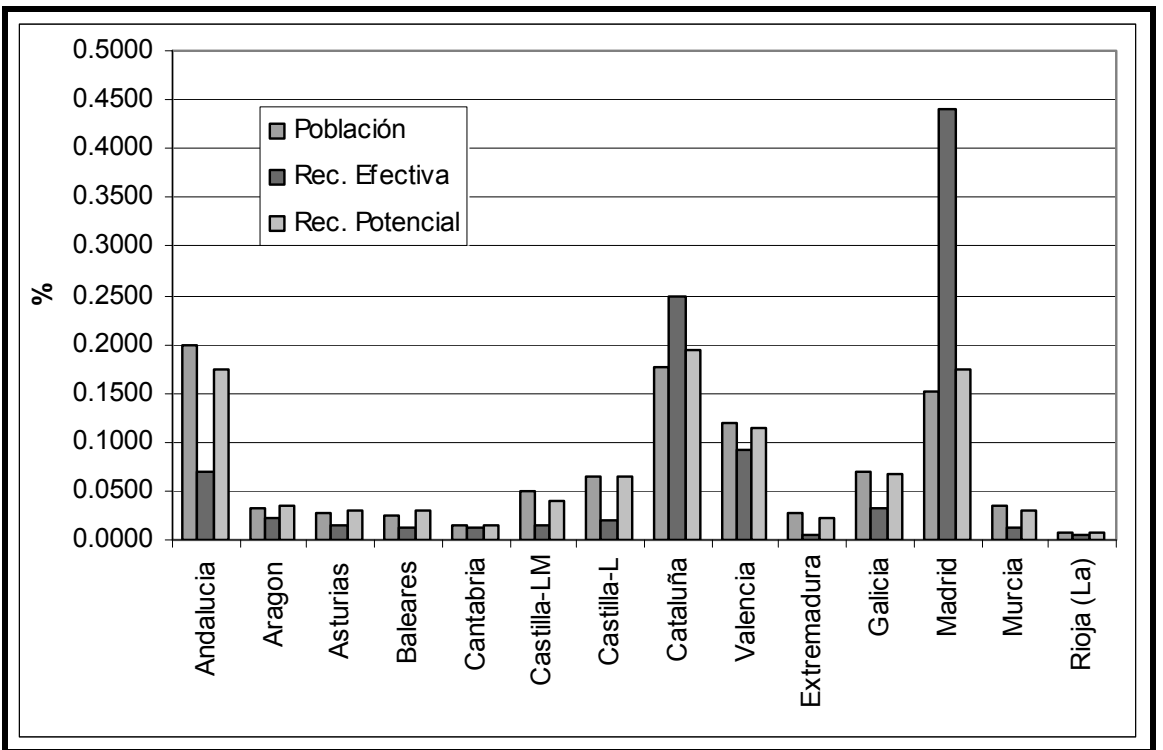

La ILUSTRACIÓN 3 muestra la existencia de una gran correlación entre población y recaudación potencial (aunque el porcentaje de participación relativo de la recaudación es mayor/menor en las CC.AA. más/menos ricas). Sin embargo la mayor parte de la recaudación efectiva se lleva a cabo, de forma muy concentrada, en unas pocas regiones.

\subsection{Recaudación por Impuestos Especiales}

Para la estimación de la recaudación potencial por los II.EE. por C.A. se ha calculado la proporción del consumo territorializado en la suma de los epígrafes 02 (Bebidas alcohólicas, tabaco y narcóticos), 04 (Vivienda, electricidad, gas y carburantes) y 07 (Compra de Vehículos, Utilización de Vehículos personales y Servicios de transporte) que forman ampliamente la base imponible de los distintos impuestos. El CUADRO 6 resume las principales cifras para 2005. 
CUADRO 6

Recaudación efectiva y potencial por los IIEE por CC.AA., 2005 (millones de euros).

\begin{tabular}{|l|r|r|r|r|}
\hline & $\begin{array}{c}\text { Recaudación } \\
\text { efectiva }\end{array}$ & $\begin{array}{c}\text { Recaudación } \\
\text { potencial }\end{array}$ & $\begin{array}{c}\text { \% Diferencia } \\
\mathbf{2 0 0 5}\end{array}$ & $\begin{array}{c}\text { \%Diferencia } \\
\text { promedio 00-05 }\end{array}$ \\
\hline Andalucía & 588,5 & $2.913,4$ & 20,20 & 17,30 \\
\hline Aragón & 6,6 & 508,1 & 1,30 & 4,41 \\
\hline Asturias & 125,4 & 426,4 & 29,40 & 28,15 \\
\hline Baleares & 16,4 & 820,4 & 2,00 & 3,82 \\
\hline Canarias & 64,5 & $1.146,0$ & 5,63 & 5,92 \\
\hline Cantabria & 51,8 & 223,4 & 23,20 & 22,93 \\
\hline Castilla-LM & 303,1 & 649,3 & 46,68 & 42,10 \\
\hline Castilla-L & 68,3 & 947,8 & 7,20 & 8,60 \\
\hline Cataluña & $1.091,3$ & $3.118,7$ & 34,99 & 35,65 \\
\hline Valencia & 183,6 & $1.977,6$ & 9,28 & 17,57 \\
\hline Extremadura & 5,8 & 329,6 & 1,76 & 3,23 \\
\hline Galicia & 63,2 & $1.085,6$ & 5,83 & 7,62 \\
\hline Madrid & $14.911,0$ & $2.856,6$ & 521,99 & 506,51 \\
\hline Murcia & 117,7 & 497,8 & 23,64 & 15,65 \\
\hline Rioja (La) & 13,2 & 109,6 & 12,07 & 12,30 \\
\hline & $17.610,3$ & $17.610,3$ & & \\
\hline
\end{tabular}

En dicho cuadro se refleja que es Madrid (506,5\%) la única C.A. donde se recauda más tributos por el concepto de IIEE que el consumo que representan. Nuevamente Andalucía es la C.A. mas perjudicada en términos absolutos con una pérdida de recaudación de 2.324,9 millones de euros.

La ILUSTRACIÓN 6 resulta muy explícita. Aunque la recaudación potencial prácticamente coincide con el ratio de población, la práctica totalidad de la recaudación por estos impuestos se lleva a cabo en una sola C.A. 


\section{ILUSTRACIÓN 4}

Recaudación efectiva y potencial por II.EE. por CC.AA., 2005.

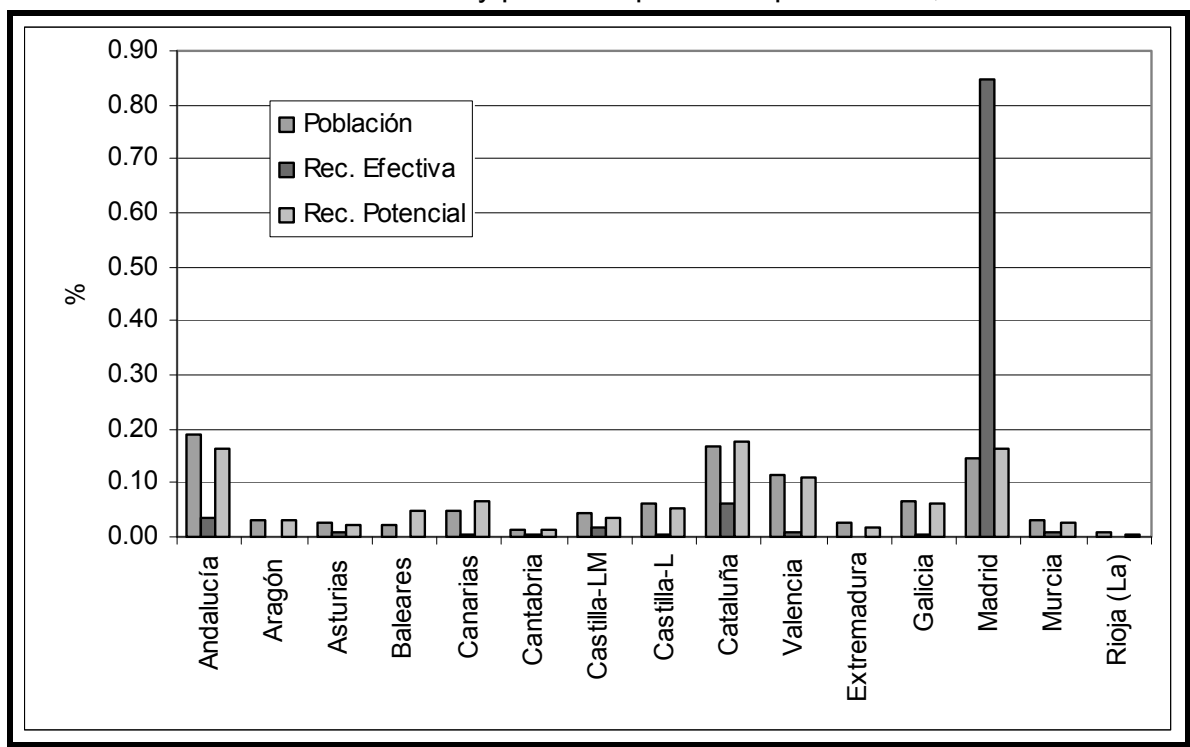

\section{EL ÍNDICE DE DESIGUALDAD TRIBUTARIA}

Los resultados obtenidos de los índices de concentración de la Recaudación Efectiva y Potencial de cada tributo se han obtenido mediante la aplicación de [4] a los paneles de recaudación efectiva y potencial de cada C.A. El Índice de Desigualdad Tributaria (TI) es la diferencia entre los dos anteriores.

En términos gráficos, la ILUSTRACIÓN 5, que recoge las curvas de Lorenz para 2005 (IVA e IIEE) y 2007(IRPF), permite apreciar que la similitud es mayor en el caso del IRPF. En el caso del IVA la desigualdad crece y, en el caso de los IIEE la desigualdad es aún mayor. 


\section{ILUSTRACIÓN 5}

Curvas de concentración de la recaudación IRPF (año 2007).

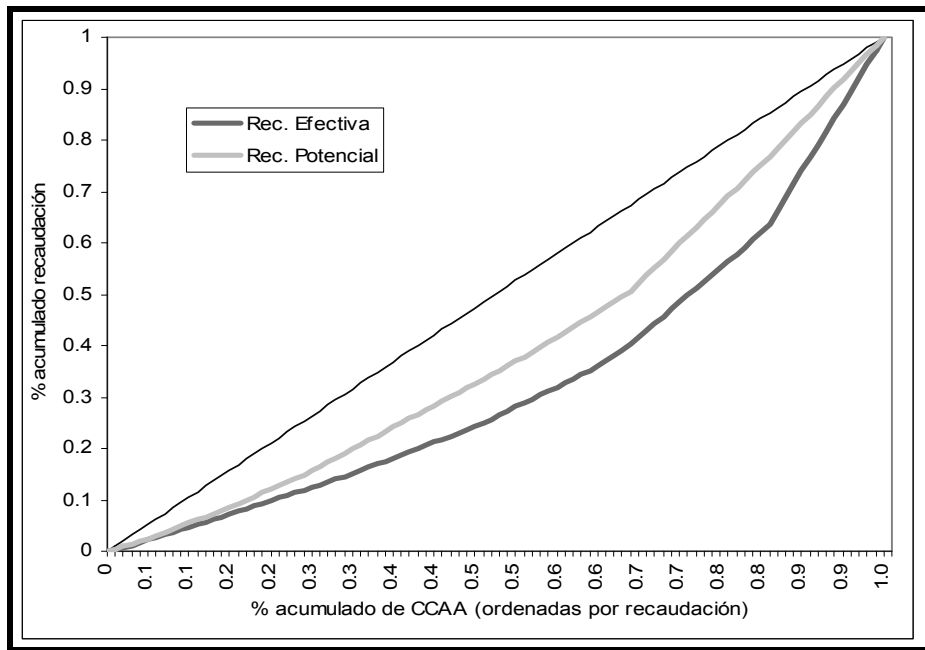

IVA (año 2005).

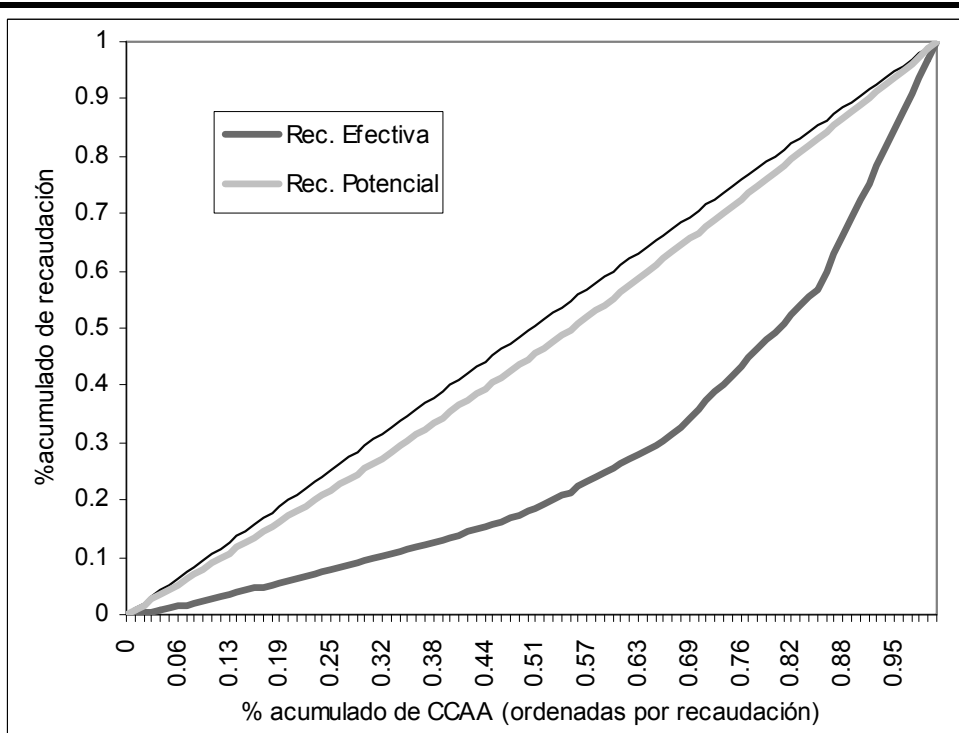


IIEE (año 2005).

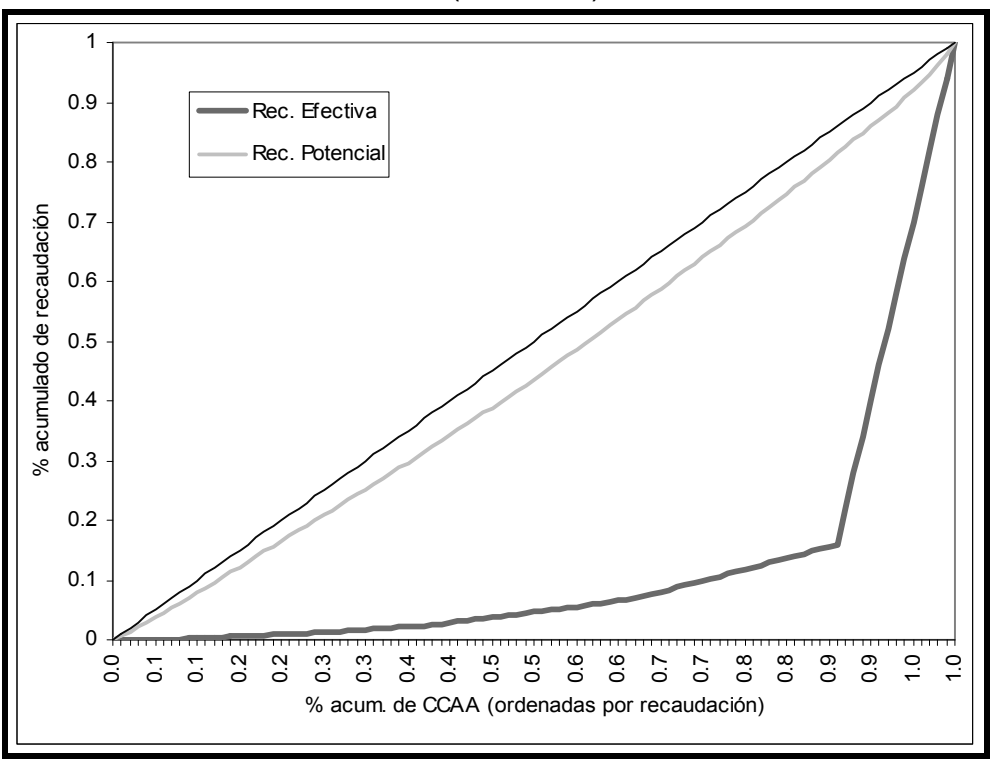

El CUADRO 7 recoge los datos estimados para los últimos años de la muestra ${ }^{5}$. Del mismo se desprende que la recaudación por el IRPF es la que presenta un patrón de comportamiento más homogéneo, la desigualdad oscila entre el 12,85\% (en 2002) y el 15,26\% (en 2000).

CUADRO 7

Índices de Concentración y de desigualdad Tributaria en España (2000 2007).

\begin{tabular}{|l|c|c|c|}
\hline & IC $_{\text {IRPF }}$ efectivo & IC $_{\text {IRPF }}$ potencial & TI IRPF \\
\hline 2000 & 38,05 & 22,79 & 15,26 \\
\hline 2001 & 35,45 & 21,92 & 13,54 \\
\hline 2002 & 34,34 & 21,49 & 12,85 \\
\hline 2003 & 34,76 & 21,24 & 13,53 \\
\hline 2004 & 36,37 & 21,44 & 14,93 \\
\hline 2005 & 33,80 & 21,26 & 12,54 \\
\hline 2006 & 33,89 & 21,47 & 12,42 \\
\hline 2007 & 33,67 & 20,59 & 13,09 \\
\hline
\end{tabular}

\footnotetext{
${ }^{5}$ Para su comparación con los IIEE se representar los resultados de los tres impuestos a partir de 2000.
} 
CUADRO 7 (Continuación)

Índices de Concentración y de desigualdad Tributaria en España (2000 2007).

\begin{tabular}{|c|c|c|c|}
\hline & IC $_{\text {IVA }}$ efectivo & IC $_{\text {IVA }}$ potencial & TI IVA \\
\hline 2000 & 49,28 & 7,41 & 41,87 \\
\hline 2001 & 47,21 & 7,02 & 40,19 \\
\hline 2002 & 46,33 & 6,81 & 39,52 \\
\hline 2003 & 44,91 & 6,49 & 38,42 \\
\hline 2004 & 44,06 & 6,37 & 37,69 \\
\hline 2005 & 44,36 & 6,32 & 38,05 \\
\hline & IC $_{\text {IIEE }}$ efectivo & IC $_{\text {IIEE }}$ potencial & TI IIEE \\
\hline 2000 & 70,84 & 11,53 & 59,31 \\
\hline 2001 & 71,98 & 11,19 & 60,78 \\
\hline 2002 & 76,90 & 11,18 & 65,72 \\
\hline 2003 & 77,46 & 10,52 & 66,94 \\
\hline 2004 & 76,01 & 9,69 & 66,32 \\
\hline 2005 & 75,21 & 9,21 & 66,01 \\
\hline
\end{tabular}

Respecto a la recaudación por el IVA, esta presenta una mayor desigualdad entre el Î́ndice de Concentración de la recaudación efectiva respecto de la potencial. El índice de desigualdad es mínimo en 2004, con un 37,69\%, y máximo en 2000 , con un $41,87 \%$.

La recaudación por los IIEE es la que presenta una mayor desigualdad. De hecho el índice de concentración de la recaudación real supera el 70\% de desigualdad en todos los casos mientras que el índice de concentración de recaudación potencial oscila siempre, con una suave tendencia a la baja, alrededor del $10 \%$.

Parece que la desigualdad en la recaudación potencial tiende a reducirse en los tres impuestos mientras que la desigualdad en la recaudación efectiva crece en el caso de los IIEE y se reduce en las otras dos figuras impositivas. 


\section{ILUSTRACIÓN 6 \\ Evolución de los Índices de Desigualdad de la Recaudación para los tres tributos (2000-2007).}

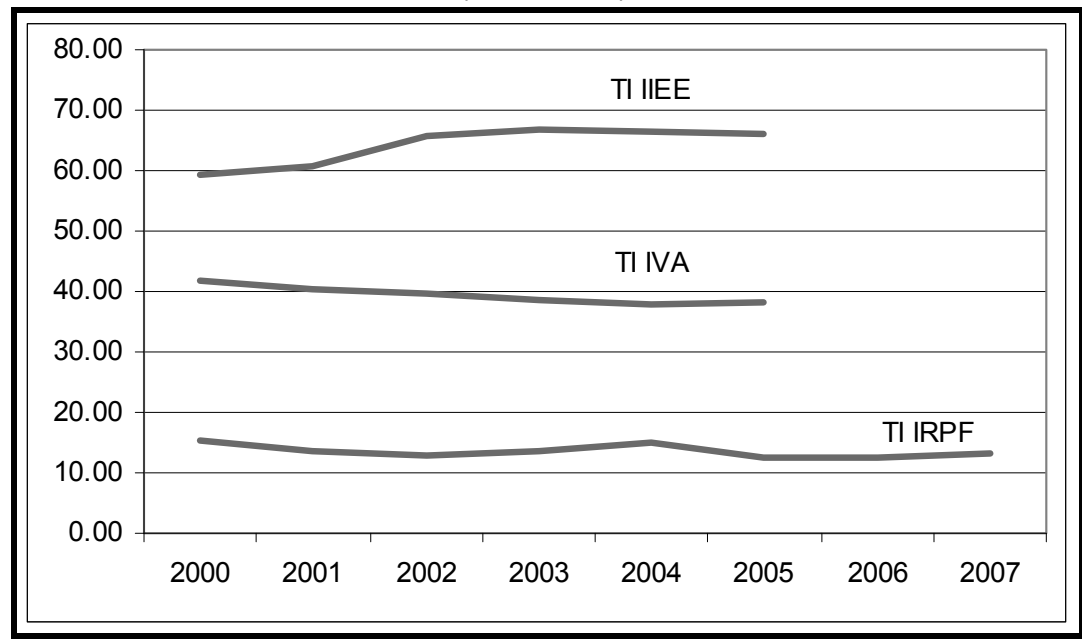

Según se desprende del CUADRO 7, hasta un 13,09\% de los recursos que reciben algunas CC.AA. por IRPF debería ser redistribuido entre el resto de CC.AA. como "compensación" por un exceso de recaudación sobre la que les corresponde en función de su actividad económica. Estas cantidades deberían ser mayores en el caso del IVA y de los IIEE aunque, en estos casos, ya se ha contemplado en la norma la distribución en función de la recaudación potencial y no la efectiva.

\section{CONCLUSIONES}

El acuerdo de financiación en vigor, con el objetivo de profundizar en la autonomía y la corresponsabilidad fiscal, amplía el espacio fiscal de las CC.AA. La ampliación del espacio fiscal implica que las CC.AA. pasan a financiarse en mayor medida de recursos tributarios generados en sus territorios y en menor medida de transferencias desde la unidad central de gobierno. Además de otros impuestos ya cedidos completamente, los impuestos en que se incrementa la descentralización son: IRPF, IVA y algunos impuestos especiales (II.EE.).

La distribución de los recursos tributarios entre las distintas CC.AA. se hace en función de distintos criterios. En el caso del IVA e II.EE. se realiza en función de índices de consumo regionales generales o índices de consumo concretos de algún bien o servicio. En el caso del IRPF la distribución se realiza en función de la cuota íntegra ingresada por los residentes en cada territorio.

La recaudación por cada impuesto en cada territorio puede diferir de la generada por las variables económicas obtenidas en dicho territorio. Existen frecuentes causas que provocan que los sujetos pasivos de cada impuesto ingresen la cuota 
tributaria en una CC.AA. distinta de aquella en que se produjo el hecho imponible y entre ellas destaca la configuración formal del dichas figuras impositivas centrada en el domicilio del sujeto pasivo.

En el caso del IVA e II.EE. la distribución que se realiza en el seno del sistema de financiación puede considerarse apropiada, en el sentido de que se aproxima más a la capacidad tributaria de cada región que la recaudación efectiva. Sin embargo, en el caso del IRPF la distribución no se ajusta por indicador económico alguno sino que el tramo autonómico se adjudica automáticamente a la región en que se realiza el ingreso.

En el caso del importe recaudado por el IRPF, se ha cuantificado que, como mínimo, un $14 \%$ del mismo, 10.669 millones de euros, se ingresan en una región distinta de la que se ha producido el hecho imponible gravado. Dado que se ha cedido un 50\% de la recaudación del impuesto, 5.334 millones de euros anuales se están transfiriendo en exceso a unas CC.AA. y en defecto a otras. En el caso de la C.A. de Andalucía, la C.A. más perjudicada en términos absolutos, la pérdida de financiación anual de recaudación asciende a 2.175 millones de euros anuales.

Dado que el IRPF es, en términos recaudatorios ${ }^{6}$, la mayor figura tributaria española, la cesión del $50 \%$ de la recaudación efectiva está provocando un importante efecto distorsionador en algunas CC.AA., que ingresan por este concepto menos recursos de los que les corresponden. Sorprende este hecho cuando las cesiones de la recaudación de IVA e IIEE se ajustan a la recaudación potencial. Este hecho nos llevaría a cuestionar esta cesión del IRPF, en los términos en los que actualmente se realiza. Además, es más relevante por cuanto es el IRPF el impuesto que mejor está soportando los ciclos económicos 7 .

Estas cantidades deberían ser redistribuidas al margen de los fondos de solidaridad que contempla el sistema de financiación como mecanismos de cierre del sistema, puesto que su finalidad es la de compensar los recursos que deberían recaudar las CC.AA. y no equipararlas por su peor situación económica.

Todo ello pone de manifiesto que para que el modelo sea justo y equitativo ha de contemplar dos tipos de transferencias compensatorias, unas denominadas por la eficiencia, que vienen a equilibrar los desajustes entre la recaudación potencial y la recaudación efectiva, y otras transferencias por equidad que vienen a equilibrar el diferencial económico entre las comunidades.

\footnotetext{
${ }^{6}$ Según los datos facilitados por el Gobierno en la presentación de los Presupuestos Generales del Estado (PGE) para el 2010, la previsión de liquidación del presupuesto de ingresos para el 2009 es que el IRPF va a suponer el $45,4 \%$ de los ingresos tributarios, porcentaje claramente superior a la siguiente figura tributaria, ya que el IVA va a suponer el 20,3\% de éstos.

${ }^{7}$ En los propios PGE para el 2010 está previsto que el IRPF disminuya un 15,1\% en el ejercicio del 2009 mientras que en el IVA su disminución se situará en el 45,0\%.
} 


\section{REFERENCIAS BIBLIOGRÁFIC.A.S}

BARBERÁN, R. (2004): "Las balanzas fiscales regionales: inventario de divergencias”. Papeles de Economía Española, 99, pp. 40-76.

BRANDFORT, D.F.; OATES, W.E. (1971): "The analylis of revenue sharing in a new approach to collective fiscal decisions". Quarterly journal of economics, 85, 416-439.

BREUSCH, T.; PAGAN, A. (1979): "A simple test for heterocedascity and random coefficient variation". Econometrita, 47, 1287-1294.

C.A.STELLS, A.; BARBERÁN, R.; BOSCH, N.; ESPASA, M.; RODRIGO, F. y RUIZHUERTA, J. (2000): Las balanzas fiscales de las Comunidades Autónomas (19911996). Análisis de los flujos fiscales de las Comunidades Autónomas con la Administración Central. Ariel Barcelona.

DICKEY, G.E.P.; FULLER, W.A. (1979): "Distribution of the estimators for autoregressive time series with a unit root". Journal of the American Statistical Association, 74, 427-431.

DE LA FUENTE, A. (2001): "Un poco de aritmética territorial: Anatomía de una balanza fiscal para las regiones españolas". Estudios de Economía Española, 91. FEDEA. Madrid.

ELLIOT, G.; ROTHENBERG, T.; STOCK, J.H. (1996): "Efficient test for an autoregresive unit root". Econometrica, 64, 813-836.

ENGLE, R.F.; GRANGER, C.W.J. (1987): "Co-integration and error-correction: Representation, estimation and testing". Econometrica, 55, 251-276.

HAUSMAN, J. (1978): "Specification test in econometrics", Econometrica, 46, pp 12511271.

HINES, J.R. y THALLER, R.H. (1995): "The flypaper efect", Journal of economics perspectives, vol. 9. $n^{\circ}$ 4, pp. 217-226.

KAKWANI, N.; WAGSTAFF, A.; VAN DOORSLAER, E. (1997): "Socioeconomic inequalities in health: Measurement, computation an statistical inference". Journal of econometrics, 77, 87-103.

QUIGLEY, J.M.; SMOLENSKY, E. (1993): "Conflicts among levesl of government in a Federal Sistem: The flipaper Efect”. Public finance. (sup), 202-215.

URIEL, E. (2001): "Análisis de la incidencia regional de los ingresos y gastos de la Administración Pública Central”, en González-Páramo (ed.): Bases para un sistema estable de financiación autonómica, Madrid: Fundación BBVA, pp. 109-378.

VAN DOORSLAER, E.; WAGSTAFF, A.; BLEICHRODT, H.; C.A.LONGE, S.; GERTHAM, U.G.; GERFIN, M.; GEURTS, J.; O'DONNELL, O.; PROPPER, C.; PUFFER, F.; RODRÍGUEZ, M.; SUNDBERG, G.; WINKELHAKE, O. (2000a): "Income-related inequalities in health: some international comparisons". Journal of Health Economics, 16, 93112.

VAN DOORSLAER, E.; WAGSTAFF, A.; VAN DER BURG, H.; CHRISTIANSEN, T.; DE GRAEVE, D.; DUVHESNE, I.; GERTHAM, U.; GERFIN, M.; GEURTS, J.; GROSS, L.; HAKKINEN, U.; JOHN, J.; KLAVUS, J.; LEU, R.; NOLAN, B.; O'DONNEL, O.; PROPPER, C.; PUFFER, F.; SCHELLHORN, M.; SUNDBERG, G.; WINKELHAKE, O. (2000b): "Equity in the delivery of health care in Europe and the US". Journal of Health Economics, 19, 553-583.

WAGSTAFF, A.; VAN DOORSLAER, E.; BURG, H.; C.A.LONGE, S.; CHRISTIANSEN, T.; CITONI, G.; GERTHAM, U.G.; GERFIN, M.; GROSS, L.; HÄKINNEN, U.; JOHNSON, P.; JOHN, J.; KLAVUS, J.; LACHAUD, C.; LAURITSEN, J.; LEU, R.; NOLAN, B.; PERÁN, E.; PEREIRA, J.; PROPPER, C.; PUFFER, F.; ROCHAIX, L.; RODRÍGUEZ, M.; SCHELLHORN, M.; SUNDBERG, G.; WINKELHAKE, O. (1999): "Equity in the finance of health care: some further international comparisons". Journal of Health Economics, 18, 263-290. 


\section{ANEXOS}

\section{Anexo 1. Test de Cointegración}

La cointegración de las variables en los distintos modelos se ha testado mediante la metodología Engle-Granger (Engle y Granger 1987) en dos pasos: a) Determinar el orden de integración de las variables en los modelos; y b) Determinar el orden de integración de los residuos. En ambos casos el orden de integración se ha estimado mediante el método de Dickey-Fuller aumentada (ADF) (Dickey y Fuller, 1979). Los retardos en ADF se han estimado en función del test DF-GLS (Elliot et al., 1996) Max. AIC y Perron. En caso de discrepancia se subraya el retardo utilizado para mostrar que suele ser el menor. Para los residuos se muestra el menor retardo que maximizara la estacionariedad de la serie. En los cuadros se muestra el intervalo de retardos sugeridos y se subraya aquel del que se muestran los resultados.

Los test de cointegración de las variables se han realizado sobre las series temporales agregadas, no sobre el panel de datos. Se ha obtenido una serie temporal por cada variable como agregación de los datos regionales y sobre dichas variables se ha testado la cointegración. La hipótesis consiste en que si existe cointegración a un nivel agregado nacional también debe existir cointegración a un nivel de desagregación regional. Los resultados de estos tests deben ser interpretados con precaución debido a los pocos grados de libertad que deja una muestra limitada a 22 observaciones.

CUADRO 8

Modelo 1: Pruebas Aumentadas de Dickey-Fuller y test de cointegración.

\begin{tabular}{|c|c|c|c|}
\hline Variable & Retardos & ADF & Diagnóstico \\
\hline irpf & $\underline{0}-1$ & $\begin{array}{c}-1,793 \\
(0,384)\end{array}$ & $\mathrm{I}(1)$ \\
\hline remun & $\underline{6}-7$ & $\begin{array}{r}-1,317 \\
(0,621)\end{array}$ & $\mathrm{I}(1)$ \\
\hline rentas & $\underline{4}-8$ & $\begin{array}{r}-0,258 \\
(0,931)\end{array}$ & $\mathrm{I}(1)$ \\
\hline tp & $\underline{0}-1$ & $\begin{array}{r}-0,938 \\
(0,775)\end{array}$ & $\mathrm{I}(1)$ \\
\hline eeb & $\underline{0}-6$ & $\begin{array}{r}-0,570 \\
(0,877)\end{array}$ & $\mathrm{I}(1)$ \\
\hline$u 1$ & $\underline{0}-3$ & $\begin{array}{r}-5,595 \\
(0,000)\end{array}$ & $\mathrm{I}(0)$ \\
\hline
\end{tabular}


Respecto a la primera parte de la prueba el test ADF muestra que todas las variables son no estacionarias. La segunda parte de la prueba, la realizada a los residuos estimados, concluye con que estos son estacionarios y $\mathrm{I}(0)$.

\section{CUADRO 9}

Modelo 2: Pruebas Aumentadas de Dickey-Fuller y test de cointegración.

\begin{tabular}{|c|c|c|c|}
\hline Variable & Retardos & ADF & Diagnóstico \\
\hline iva & $\underline{0}-1$ & $\begin{array}{c}-1,806 \\
(0,378)\end{array}$ & $\mathrm{I}(1)$ \\
\hline cons_terr & $\underline{0}-1$ & $\begin{array}{c}-0,931 \\
(0,778)\end{array}$ & $\mathrm{I}(1)$ \\
\hline u3 & $\underline{1}-6$ & $\begin{array}{c}-3,421 \\
(0,010)\end{array}$ & $\mathrm{I}(0)$ \\
\hline
\end{tabular}

En el caso del modelo del IVA, los tres test sobre las variables indican que estas son no estacionarias de orden 1 . Respecto a los residuos, el test ADF indica que son estacionarios y I(0) al 99\% de seguridad.

\section{Anexo 2. Test de especificación}

\section{CUADRO 10}

Test de especificación.

\begin{tabular}{|lcc|}
\hline & IRPF & IVA \\
\hline \multirow{2}{*}{ LM) Breusch-Pagan $\left(X^{2}\right)$} & 1053,83 & 2376,17 \\
& $(0,0000)$ & $(0,0000)$ \\
Hausman $\left(X^{2}\right)$ & 74,67 & 2,86 \\
& $(0,000)$ & $(0,240)$ \\
\hline
\end{tabular}

\section{Anexo 3. Test de colinealidad}

\section{CUADRO 11}

Modelo 1: Test de colinealidad.

\begin{tabular}{|c|c|c|}
\hline Variable & VIF & 1/VIF \\
\hline remun & 100,34 & 0,010 \\
\hline eeb & 55,46 & 0,018 \\
\hline rentas & 16,85 & 0,059 \\
\hline tp & 14,55 & 0,069 \\
\hline dummy00 & 2,25 & 0,444 \\
\hline Media VIF & 37,89 & \\
\hline
\end{tabular}


Se dice que existe colinealidad en una variable cuando el valor VIF (factor de inflación de la varianza) es superior a 30 . El modelo de IRPF adolece de este problema que afecta a los errores estándar de los parámetros estimados. 